\title{
Latex hand glove: a safe and convenient device for the endoscopic removal of foreign bodies
}

During the endoscopic removal of a sharp or pointed object from the upper gastrointestinal tract, both the pharyngoesophageal junction and the esophagogastric junction can be easily injured. The use of a latex protector hood and overtube has been previously reported as world-standard equipment $[1,2]$. However, their costs are usually high. Furthermore, there is much patient distress associated with their use during the endoscopic examination. We therefore devised a new procedure.

The latex hand glove (LHG) is a basic necessity for every endoscopic examination. It is one of the reasonably priced endoscopic instruments ( Fig. 1). However, the other intended use of LHG has not been previously reported. We herein describe the use of the LHG for the endoscopic removal of foreign bodies.

The LHG (Toray Medical Co., Ltd.; Cat.No. $8857 \mathrm{SN}$ ) was used in the following way:

1. The finger part of the glove ( $\bullet$ Fig. 2 ) is cut out and firmly attached to the endoscope with vinyl tape ( $\bullet$ Fig. 3 ).

2. Straight grasping forceps are used as an endoscopic grasper of foreign bodies (๑ Fig. 4).

3. When the endoscope is pulled back after grasping, the LHG is automatically reversed, and foreign bodies are enveloped within ( $\bullet$ Fig. 5). The sharp and pointed objects can then be safely removed while carefully protecting the gastrointestinal mucosa.

In our hospital, this procedure has sometimes been selected for the removal of foreign bodies. The procedure has been successful and has not caused any complications. In addition, the use of the LHG device has demonstrated a good cost performance. Neither the overtube nor the latex protector hood is needed during this procedure and it is therefore also less painful for patients. These results suggest that the LHG may therefore be a convenient, safe, and useful piece of equipment, and we recommend its use for the endoscopic removal of foreign bodies.

Endoscopy_UCTN_Code_TTT_1AO_2AL

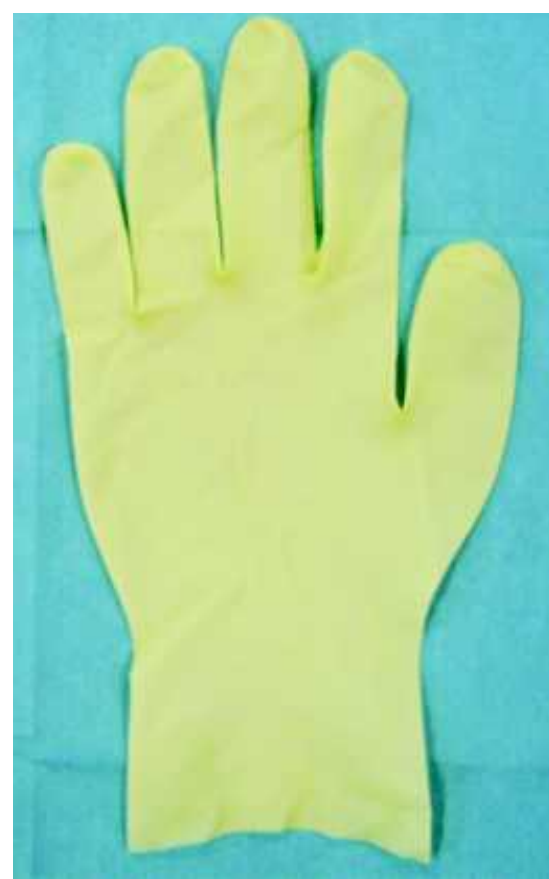

Fig. 1 A latex hand glove, which is generally used for endoscopy.

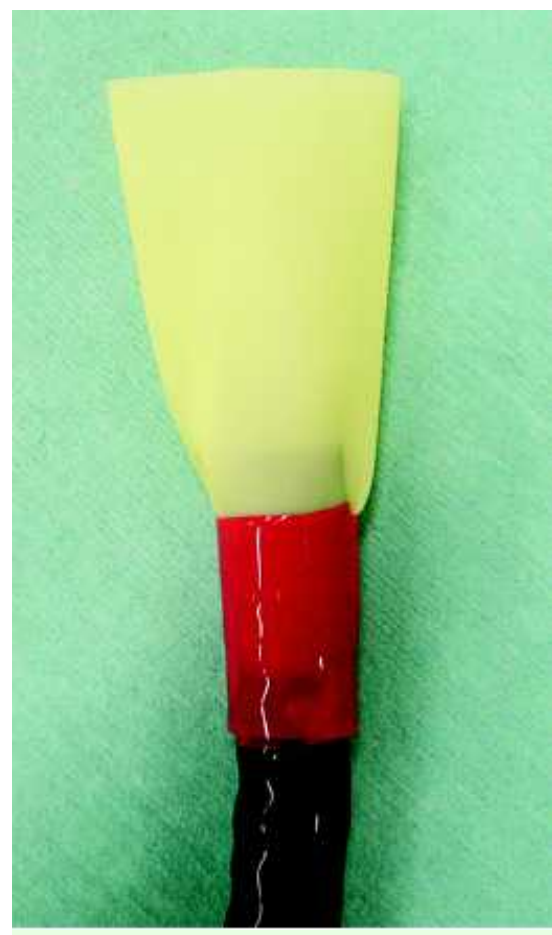

Fig. 3 The finger part of the latex hand globe is firmly attached to the endoscope with vinyl tape.

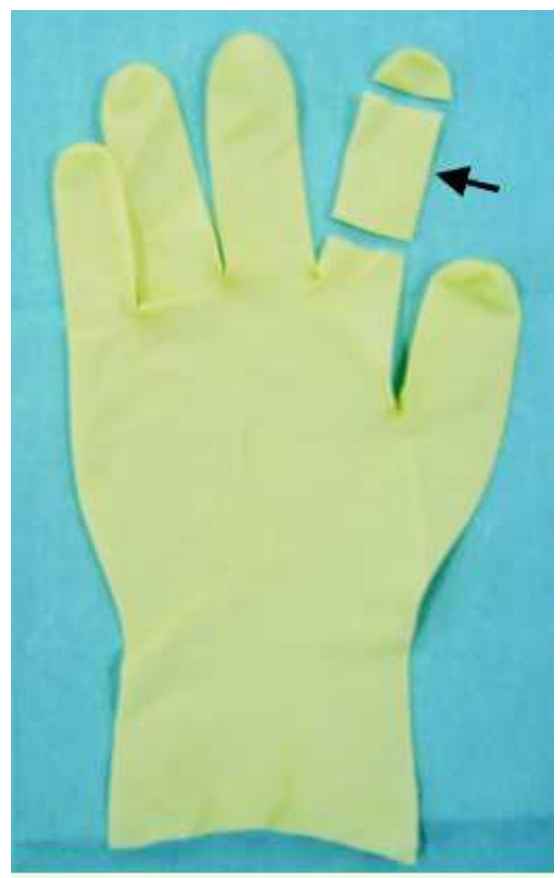

Fig. 2 The finger part is cut out of the latex hand glove.

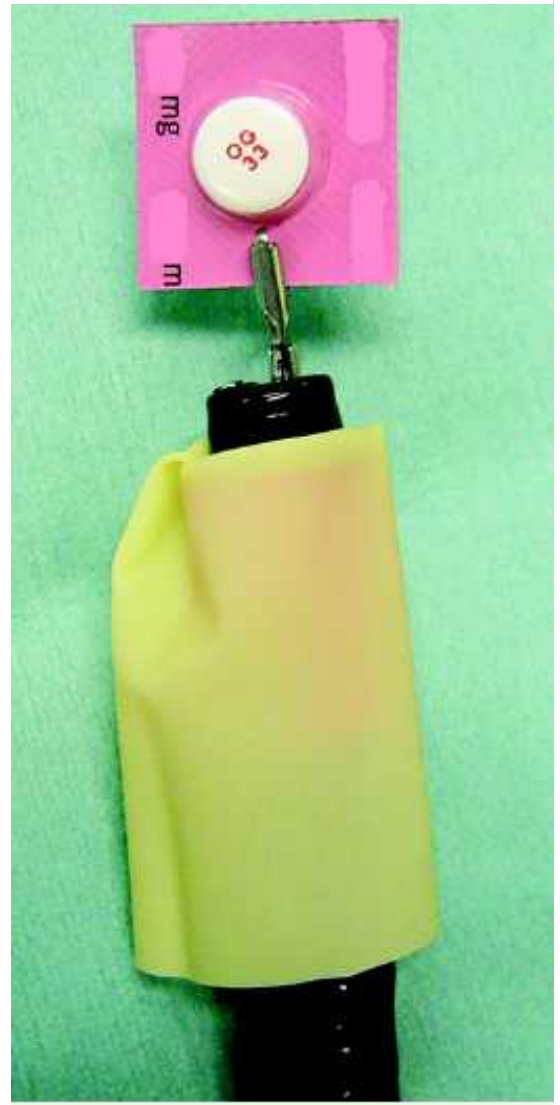

Fig. 4 A press-through package is grasped using straight grasping forceps with an endoscope. 


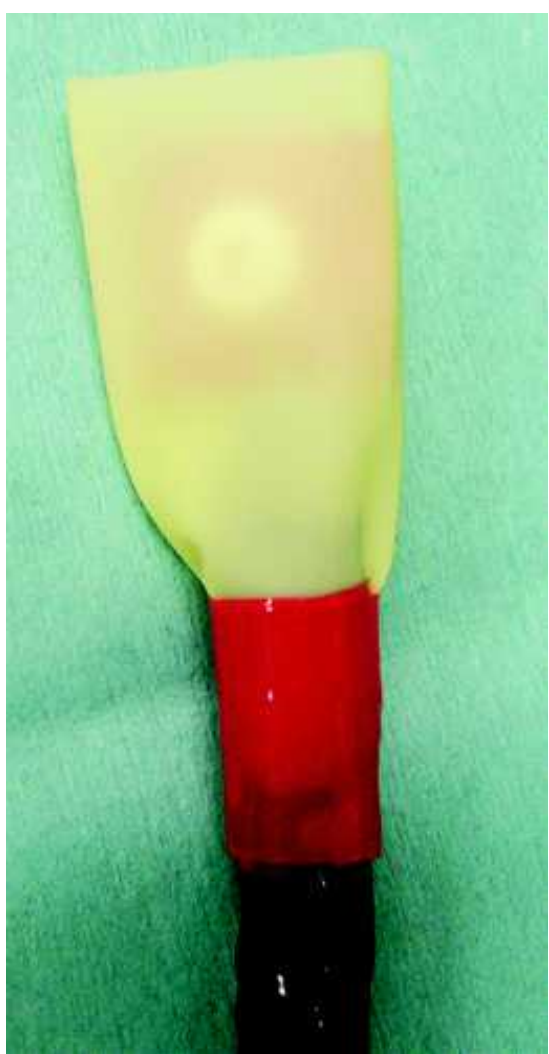

Fig. 5 The latex hand glove is automatically reversed and the press-through package is enveloped within the glove when the endoscope is pulled back.
S. Yoshida ${ }^{1}$, M. Shimada ${ }^{1}$, T. Ueno ${ }^{2}$,

A. Kato ${ }^{1}$, M. Yoshino ${ }^{1}$

Department of Gastroenterology, Internal Medicine, TMG Asakadai Central

General Hospital, Saitama, Japan

2 Critical Care Medicine, Hachioji Medical Center, Tokyo Medical University, Tokyo, Japan

\section{References}

1 Smith MT, Wong RK. Esophageal foreign bodies: types and techniques for removal. Curr Treat Options Gastroenterol 2006; 9: $75-84$

2 Bertoni G, Sassatelli R, Conigliaro $R$ et al. A simple latex protector hood for safe endoscopic removal of sharp-pointed gastroesophageal foreign bodies. Gastrointest Endosc 1996; 44: $458-461$
Bibliography

DOI $10.1055 / \mathrm{s}-2008-1077420$

Endoscopy 2008; 40: E195-E196

(c) Georg Thieme Verlag KG Stuttgart · New York . ISSN 0013-726X

\section{Corresponding author}

\section{S. Yoshida, MD, PhD}

Department of Gastroenterology Internal Medicine

TMG Asakadai Central General Hospital

1-8-10, Nishi-benzai

Asaka-shi

Saitama 351-8551

Japan

Fax: +81-48-4662735

carcinogenesis@hotmail.com 